\title{
ADSORCIÓN DE PLOMO (II) EN SOLUCIÓN ACUOSA CON TALLOS Y HOJAS DE Eichhornia crassipes
}

\section{ADSORPTION OF LEAD (II) WITH STEMS AND LEAVES OF Eichhornia crassipes IN AQUEOUS SOLUTION}

\author{
Lissette Vizcaíno Mendoza ${ }^{1}$, Natalia Fuentes Molina ${ }^{2}$, Harold González Fragozo ${ }^{3}$
}

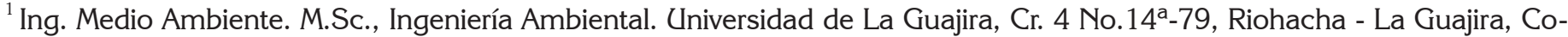
lombia, email: lvizcaino@uniguajira.edu.co; ${ }^{2}$ Ing. Ambiental y Sanitario, M.Sc. Ciencias Ambientales. Universidad de La Guajira, Cr. 9 No. 3 sur - 20, San Juan del Cesar - La Guajira, Colombia, e-mail: nnfuentes@uniguajira.edu.co; ${ }^{3}$ Microbiólogo, Esp. Pedagogía Ambiental. Instituto Nacional de Formación Técnico profesional, Cr. 13 No.7a - 61, San Juan del Cesar - La Guajira, e-mail: hgonzalez@infotep.edu.co
\end{abstract}

Rev. U.D.C.A. Act. \& Div. Cient. 20(2): 435-444, Julio-Diciembre, 2017

\section{RESUMEN}

La presencia de metales pesados, como el plomo $\left(\mathrm{Pb}^{+2}\right)$, en los cuerpos de agua genera alteraciones sobre la calidad ambiental y la salud pública, debido a su solubilidad y su capacidad de acumulación en la cadena trófica, problemática que se puede incrementar por la acumulación de Eichhornia crassipes, una maleza acuática con alta capacidad invasora, cuya presencia en los ecosistemas acuáticos favorece los procesos de eutrofización y crecimiento de microorganismos patógenos, vectores de enfermedades. Como alternativa para la eliminación de metales pesados y el aprovechamiento de tallos TEC y hojas HEC de E. crassipes, se evaluó la capacidad de adsorción y de eficiencia de remoción de $\mathrm{Pb}^{+2}$ en solución acuosa, de dicha biomasa. Inicialmente, se realizaron ensayos batch, para analizar la influencia de la dosis de adsorbente, tiempo de contacto y pH de la solución. Como método de disposición final, se analizó la calcinación, a temperaturas de 700 y $800^{\circ} \mathrm{C}$. Los datos experimentales de equilibrio fueron correlacionados, utilizando los modelos de Langmuir y Freundlich. El modelo que mejor se ajustó fue el de Langmuir, con $\mathrm{R}^{2}=0,9816$ TEC y $R^{2}=0,9854$ HEC, lográndose una máxima capacidad de adsorción de 172,41 mg/g TEC y 131,58mg/g HEC, con 0,2g de biomasa/200mL, pH 5,5 y $3 \mathrm{~h}$ de contacto. En todos los ensayos, se lograron remociones de $\mathrm{Pb}^{+2}$ superiores al $97 \%$. Los ensayos de calcinación indican que, a temperaturas $\geq 800^{\circ} \mathrm{C}$, es posible estabilizar la biomasa residual, impidiendo que los cationes metálicos removidos sean liberados de la matriz biológica, por efectos de soluciones lixiviantes de bajo $\mathrm{pH}$.

Palabras clave: Biosorción, Buchón de agua, isoterma de Langmuir, isoterma de Freundlich, metales pesados.

\section{SUMMARY}

The presence of heavy metals such as lead $\left(\mathrm{Pb}^{+2}\right)$ in water bodies causes alterations in environmental quality and public health due to their solubility and capacity of accumulation in the food chain. Problems that can be increased by the accumulation of Eichhornia crassipes an aquatic weed with high invasive capacity whose presence in the aquatic ecosystems favors the processes of eutrophication and growth of pathogenic microorganisms vectors of diseases. As an alternative for the removal of heavy metals and the use of TEC stems and $\mathrm{HEC}$ leaves of $E$. crassipes, the adsorption capacity and removal efficiency of $\mathrm{Pb}^{+2}$ in aqueous solution of this biomass were evaluated. Initially batch tests were performed to analyze the influence of the adsorbent dose, contact time and solution $\mathrm{pH}$. As final disposal method, the calcination was analyzed at temperatures of 700 and $800^{\circ} \mathrm{C}$. The equilibrium experimental data were correlated using the Langmuir and Freundlich models. The best fit model was the Langmuir model with R2 $=0.9816$ TEC and R2 $=0.9854$ HEC, achieving a maximum adsorption capacity of $172.41 \mathrm{mg} / \mathrm{g}$ TEC and $131.58 \mathrm{mg} / \mathrm{g}$ HEC with $0.2 \mathrm{~g}$ Of biomass $/ 200 \mathrm{~mL}, \mathrm{pH} 5.5$ and 3 h of contact. $\mathrm{Pb}^{+2}$ removals above $97 \%$ were achieved in all tests. Calcination tests indicate that at temperatures $\geq 800^{\circ} \mathrm{C}$ it is possible to stabilize the residual biomass by preventing the removed metal cations from being released from the biological matrix by the effects of low $\mathrm{pH}$ leaching solutions.

Key words: Biosorption, water buffer, Langmuir isotherms, Freundlich isotherms, heavy metals.

\section{INTRODUCCIÓN}

El plomo es una sustancia ambientalmente abundante, que se encuentra en las aguas marinas, con concentraciones 
entre 0,003 y $0,20 \mathrm{mg} / \mathrm{L}$, en el suelo de terrenos no cultivados, con valores de 8 a $20 \mathrm{mg} \mathrm{Pb} / \mathrm{kg}$, mientras que, en terrenos cultivados, se puede llegar a encontrar por encima de $360 \mathrm{mg} \mathrm{Pb} / \mathrm{kg}$ y cerca de fuentes de contaminación industrial, el suelo alcanza contenidos de $10 \mathrm{~g} \mathrm{~Pb} / \mathrm{kg}$ o más (Rubio et al. 2004). La contaminación del agua por plomo, se debe a los efluentes industriales no tratados, generados por fábricas de pinturas, baterías, municiones, minería, entre otros. Debido a su toxicidad, la Agencia de Protección Ambiental (EPA) lo ha clasificado entre las 20 sustancias químicas más peligrosas para la salud humana, estableciendo un límite máximo permisible de 0,001ppm, en agua potable (Lavado et al. 2010).

Los métodos de mayor aplicación en el tratamiento de efluentes que contienen metales pesados, como el plomo, son la precipitación, el intercambio iónico, la osmosis inversa y la adsorción (Lavado et al. 2010). La adsorción es un fenómeno superficial, en el cual, un soluto o adsorbato (átomos, iones o moléculas) es transferido a un material sólido poroso (adsorbente), separándolo de la fase líquida, en que se encontraban inicialmente, en contraposición a la absorción, que es un fenómeno de volumen. La mayor parte de los adsorbentes son materiales altamente porosos, donde el proceso tiene lugar fundamentalmente sobre las paredes de los poros, en puntos específicos (McCabe et al. 1998).

Cuando la adsorción se desarrolla empleando materiales de origen biológico, se denomina biosorción, la cual, es una interacción física indirecta entre iones y componentes celulares, ya sea de organismos vivos o muertos, como microorganismos, hongos, levaduras, algas, residuos vegetales, entre otros (Murithi et al. 2014); debido a la gran afinidad del adsorbente por las especies del adsorbato, este último es atraído hacia el sólido y enlazado por diferentes mecanismos. Este proceso continúa hasta que se establece un equilibrio entre el adsorbato disuelto y el adsorbato enlazado al sólido (Cuizano \& Navarro, 2008).

Eichhornia crassipes conocida como Jacinto acuático o buchón de agua es una macrofita con carácter altamente hidrofílico (Atehortúa \& Gartner, 2013), utilizada en procesos de fitorremediación, técnica en la que se aprovecha la capacidad de la planta para adsorber, acumular, metabolizar, volatilizar o estabilizar contaminantes, como los metales pesados (Delgadillo et al. 2011). Sus tallos y sus hojas constituidos por polisacáridos, como lignina, celulosa y hemicelulosa, se distribuyen en su pared celular, a nivel nano-estructural, dando como resultado redes de lignina e hidratos de carbono, cuyos principales grupos funcionales generan sobre la superficie porosa del material sitios activos, con alta afinidad, para la captura y la eliminación de metales pesados, tales como Pb(II) (Guo et al. 2007; Ramírez \& Enríquez, 2015).
Especies similares, como Salvinia biloba raddi (Tello et al. 2015) y Typha dominguensis y Canna generalis (Vera et al. 2016) han sido empleadas en procesos de fitoremediación de efluentes contaminados con plomo, logrando eficiencias de remoción, superiores al $85 \%$.

En la presente investigación, se evaluó la capacidad de adsorción y la eficiencia de remoción de $\mathrm{Pb}$ (II) de la biomasa seca de tallos y de hojas de la macrofita acuática $E$. crassipes, a fin de generar una alternativa económica y ambientalmente sostenible, para el tratamiento de efluentes contaminados con bajas concentraciones de metales pesados, en el orden de 1 a 100mg/L (Cuizano \& Navarro, 2008).

\section{MATERIALES Y MÉTODOS}

Se cosecharon tallos y hojas de $E$. crassipes ubicados en las riberas del río Ranchería, en un lugar ubicado a 930m de la Universidad de La Guajira, en jurisdicción del municipio de Fonseca, en coordenadas $10^{\circ} 53^{\prime} 59.1^{\prime \prime} \mathrm{N}$ y $72^{\circ} 49^{\prime} 47.6^{\prime \prime} \mathrm{W}$. En el laboratorio, se lavaron con agua de la llave para eliminar residuos de sedimentos y otras impurezas. Los tallos y las hojas, se cortaron en fracciones de $1 \mathrm{~cm}$ de longitud, aproximadamente, se lavaron con agua destilada y se secaron a $60^{\circ} \mathrm{C}$, hasta peso constante. Las biomasas, se trituraron, empleando una licuadora doméstica y se tamizaron para la selección de un tamaño de partícula de $1 \mathrm{~mm}$, de acuerdo con el procedimiento experimental utilizado por Atehortúa \& Gartner (2013) y Vizcaino \& Fuentes (2015).

Ensayos de biosorción de plomo en solución acuosa: Se realizaron en un sistema batch, empleando un equipo de prueba de jarras, con soluciones de concentración inicial de 30,951ppm de $\mathrm{Pb}^{+2}$, preparadas con $\mathrm{PbO}_{2}$. Los parámetros velocidad de agitación $200 \mathrm{rpm}$ y temperatura $28,5^{\circ} \mathrm{C}$, se mantuvieron constantes. Se analizó la influencia de las variables independientes: modificación alcalina, dosis de adsorbente $(\mathrm{g} / \mathrm{mL})$, tiempo de contacto $(\mathrm{h})$ y pH de solución, sobre las variables de respuesta: capacidad de adsorción $(\mathrm{mg} / \mathrm{g})$ y porcentaje de remoción (\%R) (Maldonado et al. 2012). La concentración residual de plomo, Ce de la fase acuosa, se determinó por espectrometría de absorción atómica. Los ensayos, se realizaron por triplicado en el laboratorio de química de la universidad de La Guajira. Las determinaciones de la concentración de Plomo, se desarrollaron en el Laboratorio Químico de Consultas Industriales (LQCI), de la Universidad Industrial de Santander.

Influencia de la modificación química: Se desarrolló en dos fases: inicialmente, la biomasa seca de tallos y de hojas de E. crassipes fue tratada con soluciones de $\mathrm{NaOH}$ y $\mathrm{Ca}(\mathrm{OH})_{2}$, a concentraciones $0,2 \mathrm{M}$, en tratamientos por separado, empleando $800 \mathrm{~mL}$ de solución, a pH 4,5 y $8 \mathrm{~g}$ de biomasa. 
Las biomasas tratadas, se secaron en estufa a $60^{\circ} \mathrm{C}$ hasta peso constante y se almacenaron para su posterior uso. Para analizar el efecto de la modificación química, se emplearon 0,5g de biomasa y $100 \mathrm{~mL}$ de solución (Vizcaino \& Fuentes, 2015), durante un tiempo de contacto de $24 \mathrm{~h}$ y un valor de $\mathrm{pH}=4,5$.

Influencia de la dosis de absorbente y tiempos de contacto: Las dosis de adsorbente se evaluaron empleando cinco concentraciones diferentes de tallos y de hojas de biomasa seca de $E$. crassipes $(0,2 ; 0,5 ; 0,8 ; 1,4 ; 2,0 ; \mathrm{g} / 200 \mathrm{~mL})$ y tiempos de contacto de $24 \mathrm{~h}$. Los valores iniciales y finales de $\mathrm{pH}$ se determinaron empleando un pH-metro WTW modelo 3110.

Para el análisis de la influencia del tiempo de contacto, se emplearon $0,5 \mathrm{~g}$ de biomasa, en $100 \mathrm{~mL}$ de solución metálica, con $\mathrm{pH}=4,5$ y tiempo de retención de 0,$5 ; 1,0 ; 1,5 ; 2$ y 3 h.

pH óptimo para la biosorción de $\mathrm{Pb}^{2+}$ con E. crassipes: En vasos de precipitado, se dispusieron $200 \mathrm{~mL}$ de solución de plomo, ajustando cada muestra a diferentes $\mathrm{pH} 3,5 ; 4,0 ; 4,5$; 5,0; 5,5 y 6,0, utilizando soluciones de $\mathrm{NaOH}$ y $\mathrm{HNO}_{3}$, a concentración $0,1 \mathrm{M}$. Se añadieron $0,5 \mathrm{~g}$ de biosorbente, preparado con tallos y con hojas de $E$. crassipes a cada muestra, agitando las muestras a $200 \mathrm{rpm}$, durante $24 \mathrm{~h}$, a temperatura ambiente. Una vez transcurrido el tiempo, las muestras se filtraron y se midió el pH final de las soluciones (Cárdenas et al. 2013).

Para analizar la influencia de las variables, se utilizó un diseño factorial de tres niveles: dosificación del adsorbente, $\mathrm{pH}$ y tiempo de contacto.

Isotermas de adsorción: Las curvas de isotermas, se realizaron empleando $100 \mathrm{~mL}$ de soluciones con concentraciones iniciales de plomo, en el rango de 20 a 100mg/L (García et al. 2013). A cada solución, se le agregaron $0,2 \mathrm{~g}$ de adsorbente y se ajustó el pH a 5,5 unidades (pH óptimo), manteniendo agitación constante durante $24 \mathrm{~h}$. El tratamiento de los datos experimentales del proceso de adsorcion de $\mathrm{Pb}^{+2}$, se realizó aplicando los modelos de Langmuir y Freundlich.

Calcinación de biomasa residual: Las biomasas remanentes de los ensayos de cinética de adsorción en soluciones con $100 \mathrm{ppm}$, se sometieron a calcinación en una mufla Thermolyne Modelo FB $1315 \mathrm{M}$, a 700 y $800^{\circ} \mathrm{C}$. Posteriormente, se tomó $1 \mathrm{~g}$ de la ceniza obtenida y se colocó en contacto con soluciones preparadas con ácido nítrico $\mathrm{HNO}_{3}$, con valores de $\mathrm{pH}$ de 2,5; 3,5 y 4,5 durante un tiempo de contacto de 24h, a temperatura ambiente (Díaz et al. 2003; Vizcaino \& Fuentes, 2015).

\section{RESULTADOS Y DISCUSIÓN}

Efectos de la modificación química: La biomasa TEC reportó una mayor capacidad de adsorción de $\mathrm{Pb}$ en todos los casos analizados, alcanzando su tasa máxima al modificarla con $\mathrm{CaCl}_{2}$, mientras que el $\mathrm{NaOH}$ mejoró ligeramente eficiencia de remoción de las HEC. En todos los casos, se logró eliminar más del $92 \%$ del plomo presente en la solución, consiguiendo solo un aumento del 2,4 y $2,78 \%$, para TEC- $\mathrm{CaCl}_{2}$ y HEC- $\mathrm{NaOH}$, respectivamente, en comparación con las remociones obtenidas con la biomasa sin modificación.

Los porcentajes de remoción superiores al $93 \%$ y reportados por la biomasa inerte sin pretratamiento indican que los tejidos de la planta poseen una alta capacidad de remoción de plomo y no requieren de modificación química para favorecer la captura del catión. Según Atehortúa \& Gartner (2013), se debe a su estructura macroporosa y mesoporosa, con baja área superficial y alto contenido de humedad $>90 \%$, la cual, al deshidratarse el material deja expuestas una gran cantidad de celdas o cámaras que favorecen la captura de los iones metálicos dispuestos en la solución y a la presencia de grupos funcionales característicos de la celulosa, hemicelulosa y lignina, que incrementan la cantidad de sitios activos disponibles.

Efectos de la concentración de biomasa: Los ensayos con diferentes dosis de biomasa (Figura 1a) muestran una disminución progresiva de la capacidad de adsorción, conforme aumenta la cantidad de material adicionado a la solución. La mayor capacidad de adsorción de plomo qTEC $=29,788 \mathrm{mg} / \mathrm{g}$ y $\mathrm{qHEC}=26,966 \mathrm{mg} / \mathrm{g}$, se logró empleando una dosis de $0,1 \mathrm{~g}$ de biomasa, dosis mayores generan una disminución de la capacidad de captura del plomo en solución, lo cual, se puede dar como consecuencia de las interacciones electrostáticas entre las partículas de biomasa, disminuyendo los sitios de contacto, por aglomeración de las mismas. Lo anterior indica que, a menor cantidad de biomasa mayor distancia entre partículas y, por consiguiente, mayor adsorción de cationes metálicos (Chuquilin \& Rosales, 2016).

Efectos del tiempo de contacto: Existe una correlación positiva entre la cantidad de metal removido y el tiempo de contacto con la biomasa vegetal, es decir, a medida que se incrementa el tiempo de exposición aumenta la cantidad de metal eliminado (Figura 1b). Al analizar el comportamiento de la capacidad de adsorción respecto al tiempo de contacto, se observa que ésta aumenta rápidamente durante los primeros $30 \mathrm{~min}$, eliminando más del $87 \%$ del plomo en solución. Tiempo seguido, la remoción de plomo se da a una tasa más lenta hasta lograr una remoción $\geq 99 \%$, a tiempos de contacto de $180 \mathrm{~min}$, alcanzando la concentración de equilibrio. A tiempos superiores, se puede generar un incre- 


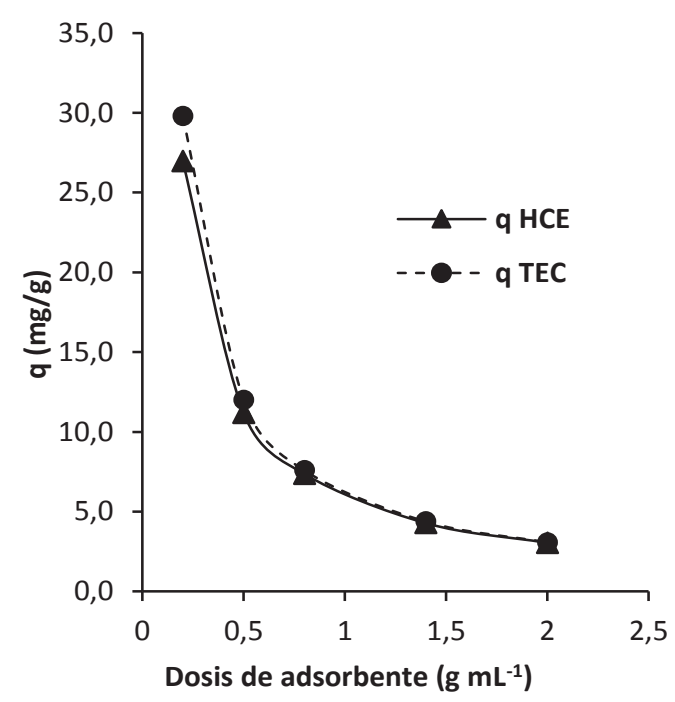

(a)

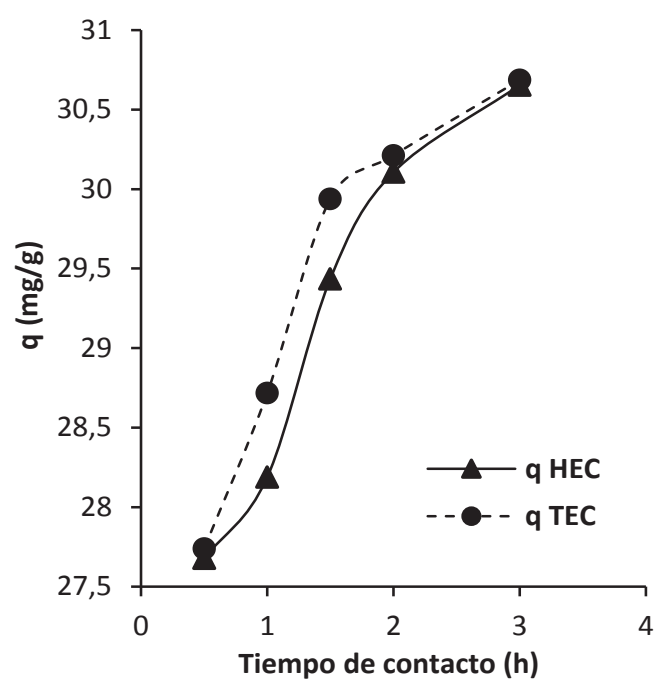

(b)

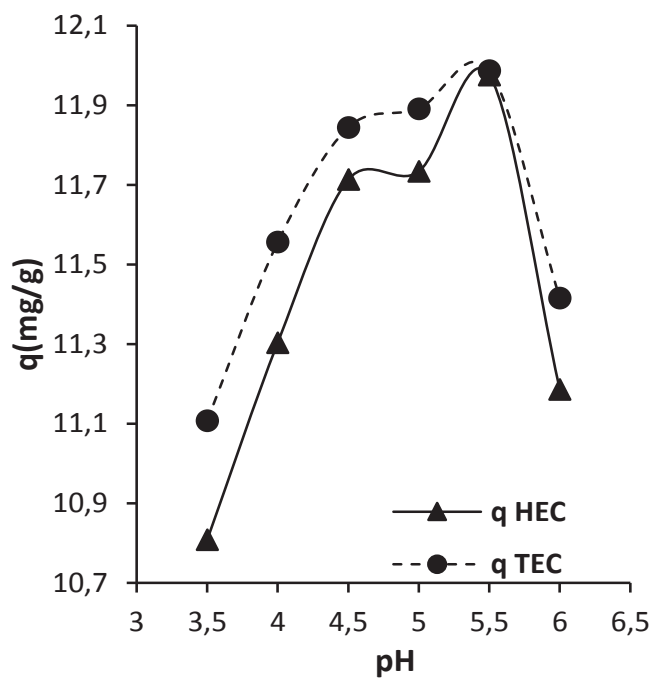

(c)

Figura 1. (a) Efectos de la concentración de absorbente; (b) tiempo de contacto y (c) pH de la solución, sobre la capacidad de adsorción de $\mathrm{Pb}^{+2}$, con hojas (qHEC) y tallos (qTEC) de Eichhornia crassipes.

mento de la concentración del metal en la solución, como consecuencia de la saturación de la superficie porosa de las biomasas con cationes de $\mathrm{Pb}^{+2}$.

Las eficiencias de remoción respecto al tiempo de contacto obtenidas con $E$. crassipes son superiores a las referenciadas por Tello et al. (2015) utilizando Salvina biloba raddi, quienes reportan remociones superiores a $90 \%$, para tiempos de contacto de $24 \mathrm{~h}$ y concentraciones iniciales de 5 , 10 y 20ppm; y a las publicadas por Vera et al. (2016), en experimentos tipo batch con Typha dominguensis y Canna generalis, en los cuales, se obtuvieron remociones de plomo superiores a $90 \%$, a las $21 \mathrm{~h}$ de contacto y alcanzando la mayor remoción a las $93 \mathrm{~h}$ de contacto, para concentraciones iniciales de 5, 10 y $15 \mathrm{mg} / \mathrm{L}$.

Efectos del pH de la solución: En figura 1c, se muestra la relación entre la capacidad de adsorción y el pH de la solución. El comportamiento de la curva indica que existe una mayor atracción entre los iones de $\mathrm{Pb}^{+2}$ y los grupos funcionales superficiales de TEC y HEC a valores de $\mathrm{pH}$, en el rango 5,0 a 6,0 unidades, obteniéndose adsorciones máximas de qTEC $=29,971 \mathrm{mg} / \mathrm{g}$ y $\mathrm{qHEC}=29,941 \mathrm{mg} / \mathrm{g}$, al pH 5,5. 
En las curvas, se observa claramente que los pH ácidos reducen la capacidad del biomaterial para capturar y retener el plomo, como consecuencia de la solubilización del catión en la matriz acuosa y la hidrólisis del material biológico. A $\mathrm{pH}$ menores a 5,5, las cargas superficiales totales se vuelven positivas, generando una competencia entre $\mathrm{H}^{+}$y $\mathrm{Pb}^{+2}$, consecuencia de una fuerza de repulsión que se forma entre ambos (Lodeiro et al. 2006) e inhibe la aproximación de los cationes metálicos.

A pH alcalinos superiores a 5,5, se forma $\mathrm{Pb}(\mathrm{OH})_{2}$, generando la precipitación del metal (Lavado et al. 2010), lo cual, impide que los sitios de contacto en la superficie de la biomasa se enlacen con la especie química, inhibiendo el proceso de biosorción.

El efecto del pH evidenciado en el proceso experimental, se puede explicar en términos de $\mathrm{pH}$ del punto de carga cero (pHpzc), en el que la superficie del biosorbente es neutra. Cuando la biomasa se mantiene en una solución que tiene pH menor que pHpzc de biomasa, la protonación de ciertos grupos funcionales ocurre y la biomasa se comporta como una polimatriz cargada positivamente. Un aumento del $\mathrm{pH}$ por encima de este punto hace que los grupos funcionales en el tipo de biomasa se desprotonen y actúen como especies negativas (Abdel-Ghani \& El-Chaghaby, 2014).
Eficiencias de remoción: En la tabla 1, se observa que el porcentaje de plomo removido con respecto a la concentración inicial $\mathrm{C}_{\mathrm{i}}=30,951 \mathrm{ppm}$ es alto para todos los ensayos analizados, alcanzando eficiencias máximas superiores al 96\%, para valores de pH 5,5 y 99\%, para tiempos de retención de 1,5h.

En cuanto al efecto de la dosis de adsorbente, los resultados obtenidos muestran que, a mayor dosis de biomasa mayor eficiencia de remoción de $\mathrm{Pb}^{+2}$, alcanzando un porcentaje superior al $97 \%$, al emplear concentraciones $\geq 0,4 \mathrm{~g} / 100 \mathrm{~mL}$. Este incremento está relacionado con el aumento del área de contacto del sólido adsorbente y el número de los sitios de biosorción, disponibles en su superficie.

Las eficiencias de remoción de $\mathrm{Pb}^{+2}$ son similares a las reportadas por Óre et al. (2015): $97 \%$, con $4 \mathrm{~g} / \mathrm{L}$ de marlo de maíz (Zeas mays); por Ramírez \& Enríquez (2015): 55\%, con $1 \mathrm{~g}$ de lignina (extraída de seudotallo de plátano) hidratada con $\mathrm{NaNO}_{3}$ 0,01M; por Atehortúa \& Gartner (2013): 86,5 y $84,08 \%$ empleando $2 \mathrm{~g} / \mathrm{L}$ de $E$. crassipes sin modificar y con modificación térmica $200^{\circ} \mathrm{C}$ por $6 \mathrm{~h}$, respectivamente y por Cárdenas et al. (2013): 100 y 94,1\% con $1 \mathrm{~g} / 100 \mathrm{~mL}$ de las cepas fúngicas Mucor rouxii IM-80 y Mucor rouxii mutante.

El análisis factorial indica que la remoción de plomo con $E$. crassipes depende, principalmente, de la concentración de

Tabla 1. Concentraciones en equilibrio (Ce) y Eficiencias de remoción (\%R) de $\mathrm{Pb}^{+2}$ usando biomasa de tallos (TEC) y hojas (HEC) de Eichhornia crassipes, como adsorbente.

\begin{tabular}{|c|c|c|c|c|c|}
\hline Biosorbente & & Ce TEC $(\mathrm{mg} / \mathrm{L})$ & $\%$ R TEC & $\mathrm{Ce} \operatorname{HEC}(\mathrm{mg} / \mathrm{L})$ & \%R HEC \\
\hline \multirow{5}{*}{$\begin{array}{l}\text { Dosis de adsor- } \\
\text { bente }(g / L)\end{array}$} & 0,2 & 1,163 & 96,24 & 1,925 & 93,78 \\
\hline & 0,5 & 0,935 & 96,98 & 1,557 & 94,97 \\
\hline & 0,8 & 0,712 & 97,70 & 0,917 & 97,04 \\
\hline & 1,4 & 0,431 & 98,61 & 0,742 & 97,60 \\
\hline & 2 & 0,261 & 99,16 & 0,457 & 98,52 \\
\hline \multirow{5}{*}{$\begin{array}{l}\text { Tiempo de con- } \\
\text { tacto (horas) }\end{array}$} & 0,5 & 3,212 & 89,62 & 3,274 & 89,42 \\
\hline & 1 & 2,234 & 92,78 & 2,763 & 91,07 \\
\hline & 1,5 & 1,012 & 96,73 & 1,515 & 95,11 \\
\hline & 2 & 0,739 & 97,61 & 0,845 & 97,27 \\
\hline & 3 & 0,265 & 99,14 & 0,297 & 99,04 \\
\hline \multirow{6}{*}{$\mathrm{pH}$} & 3,5 & 3,183 & 89,72 & 3,931 & 87,30 \\
\hline & 4 & 2,062 & 93,34 & 2,693 & 91,30 \\
\hline & 4,5 & 1,341 & 95,67 & 1,669 & 94,61 \\
\hline & 5 & 1,223 & 96,05 & 1,617 & 94,78 \\
\hline & 5,5 & 0,984 & 96,82 & 1,012 & 96,73 \\
\hline & 6 & 2,413 & 92,20 & 2,987 & 90,35 \\
\hline
\end{tabular}


biomasa y el tiempo de contacto (Figura 2). La acidez de la solución genera una influencia significativa solo a valores de pH menores a 5,0; lo anterior indica, que el rango ideal de pH para la remoción de plomo con la biomasa utilizada está entre 5,0 y 6,0 unidades, confirmando que 5,5 es el valor óptimo tal y como se evidenció en los ensayos, a diferentes $\mathrm{pH}$.

Las relaciones concentración de biomasa y tiempo de contacto indican que la adsorción se da a una alta tasa durante los primeros 90 minutos, eliminando más del 97\% del plomo en solución acuosa. El valor del pH no genera gran influencia sobre la biomasa al incrementar su concentración en la solución, observándose que las eficiencias son cercanas al 96\%, con fluctuaciones de $\pm 1 \%$.

De acuerdo con el comportamiento de las curvas, se determina que las condiciones ideales para la remoción de plomo con tallos y hojas de $E$. crassipes se obtienen al utilizar una

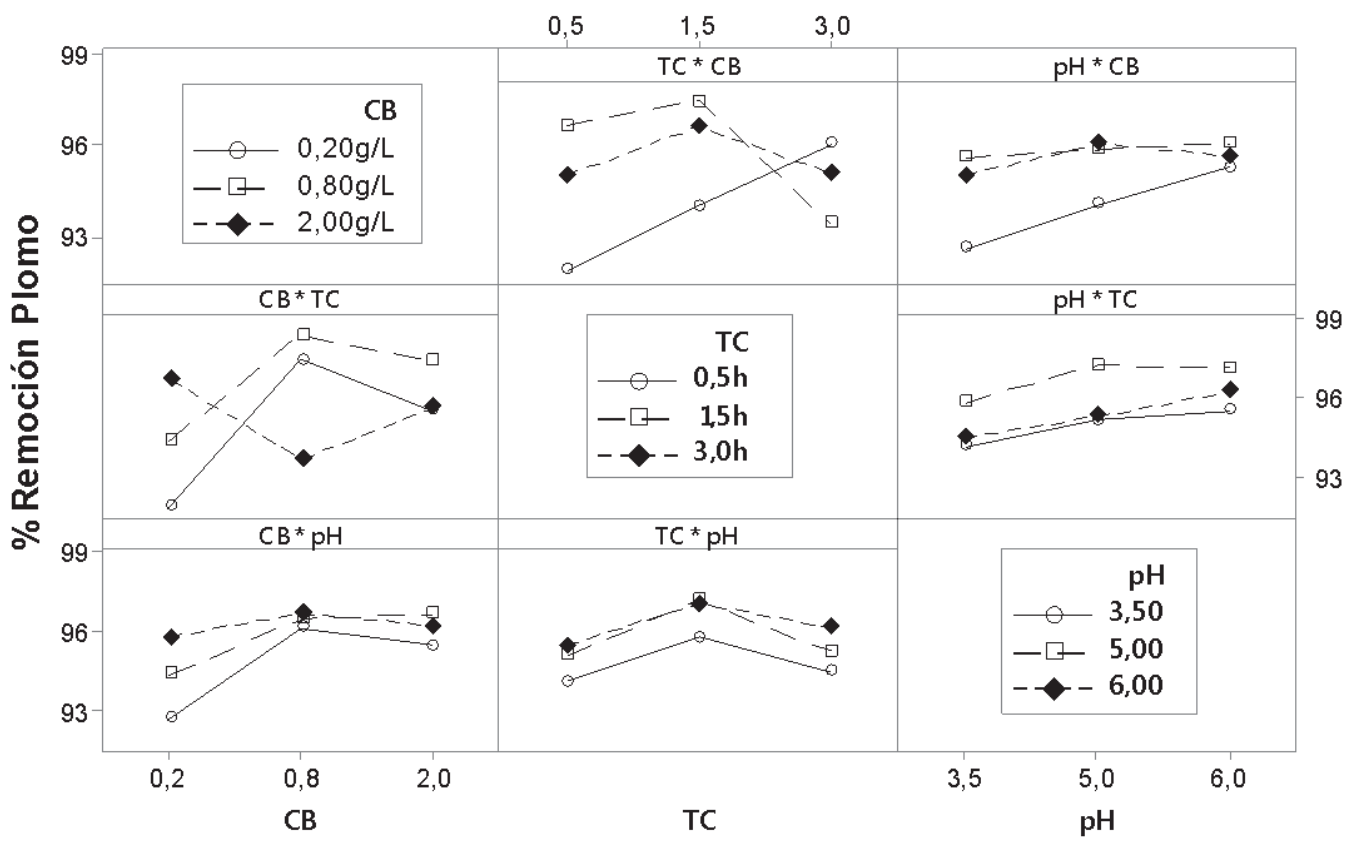

Figura 2. Interacción de las variables concentración de biomasa (CB), tiempo de contacto (TC) y pH en la remoción de plomo con Eichhornia crassipes.

concentración de $0,8 \mathrm{~g} / \mathrm{L}$ de biomasa, en una solución de $\mathrm{pH}$ ligeramente ácido, con valor óptimo de 5,5, durante un tiempo de contacto de $1,5 \mathrm{~h}$, características experimentales, para las cuales, se logra una eliminación de $\mathrm{Pb}^{+2}$ superior al $97 \%$.

Isotermas de adsorción de plomo $\left(\mathrm{Pb}^{+2}\right)$ : Los factores de correlación indican que los datos experimentales obtenidos en el proceso de adsorción de $\mathrm{Pb}^{+2}$ con hojas y tallos de $E$. crassipes se ajustan mejor al modelo de isoterma de Langmuir $R^{2}=0,9854$ y $R^{2}=0,9816$, respectivamente (Figura 3).

Los resultados obtenidos con el modelo de Langmuir indican que las biomasas de tallos y de hojas de $E$. crassipes presentan una superficie homogénea con un número específico de sitios activos que, al saturarse, inhiben el proceso de adsorción del plomo en solución. Asimismo, según este modelo (Tabla 2), las capacidades máximas de adsorción de $\mathrm{Pb}$ para tallos y para hojas son $172,4138 \mathrm{mg} / \mathrm{g}$ y $131,5789 \mathrm{mg} / \mathrm{g}$, res- pectivamente, empleando una dosis de $2 \mathrm{~g}$ biomasa/200mL solución, pH 5,5 y tiempo de contacto de $24 \mathrm{~h}$.

Se establece también, que el enlace entre los sitios activos del adsorbente y el metal se da, principalmente, por fuerzas físicas, limitándose a la formación de una monocapa, en la cual, el número de especies metálicas adsorbidas no excede el total de sitios disponibles y no se considera ninguna reacción de desorción, que pueda tener lugar durante el proceso de remoción.

Por su parte, el modelo de Freundlich indica que existe una fuerte interacción de los iones de $\mathrm{Pb}^{+2}$ con los centros activos de los biosorbentes TEC $\mathrm{Kf}=20,912$ y $\mathrm{n}=1,536$ y HEC $\mathrm{Kf}=19,747$ y $\mathrm{n}=1,627$ ), mientras que el análisis de los valores de $R_{\mathrm{L}}$ indican que existe un comportamiento isotérmico favorable de las biomasas $0<R L<1$. 
Isoterma de Langmuir HEC

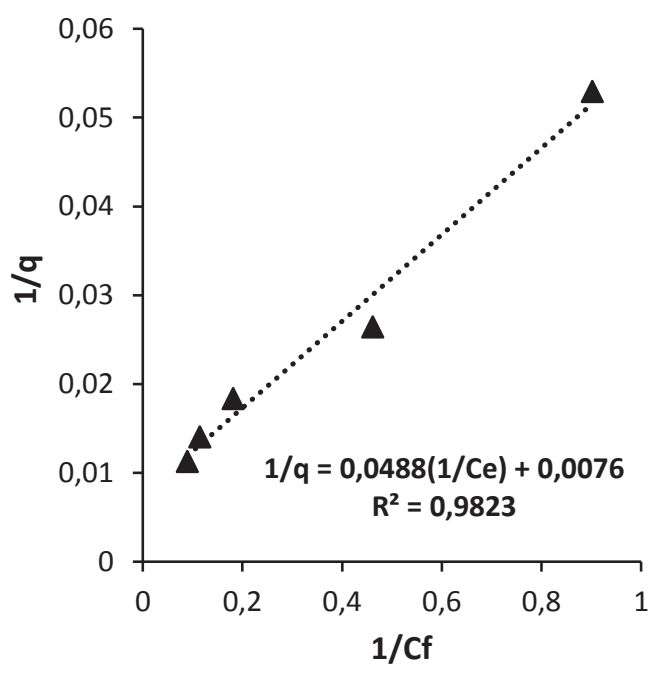

Isoterma de Freundlich HEC

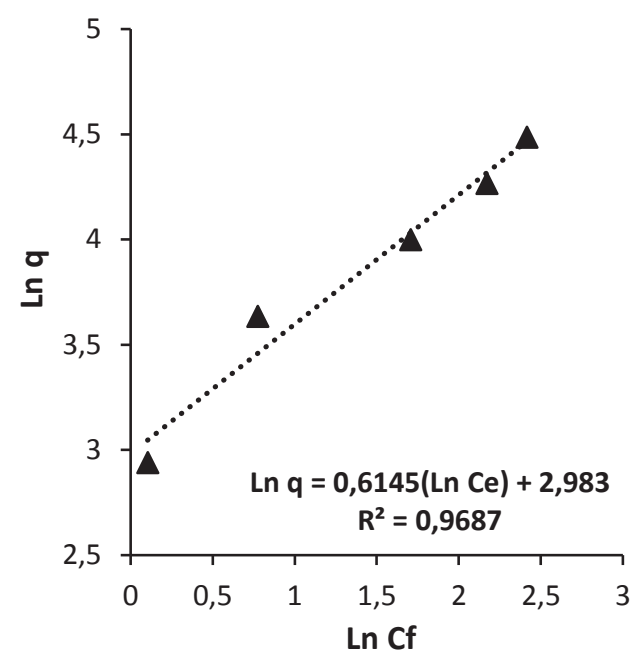

Isoterma de Langmuir TEC

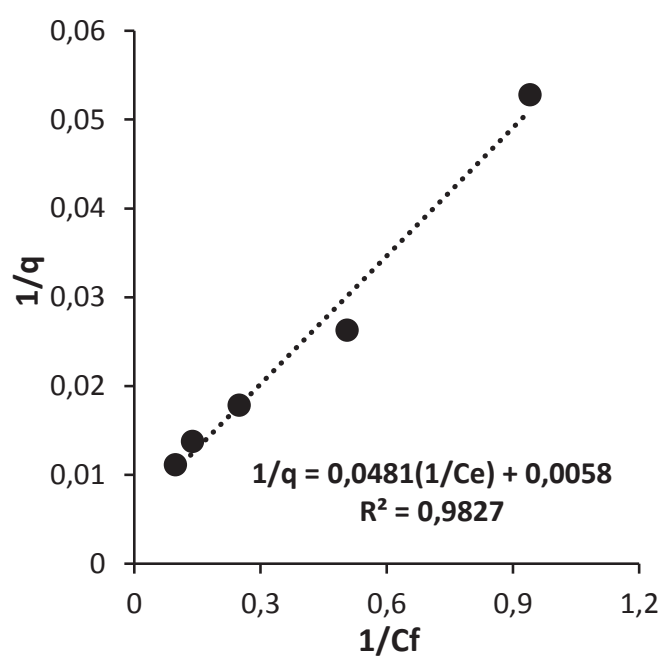

Isoterma de Freundlich TEC

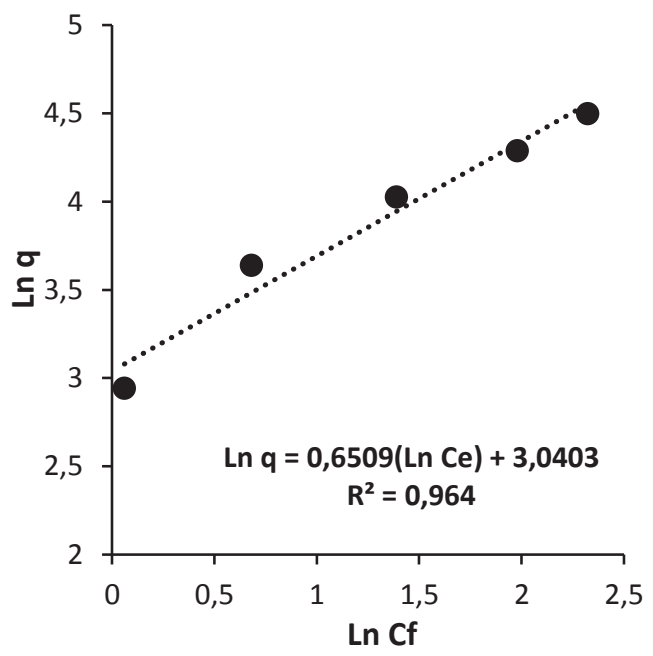

Figura 3. Modelo de isotermas de adsorción de Pb (II), con tallos (TEC) y hojas (HEC) de Eichhornia crassipes.

Tabla 2. Constantes de Langmuir y Freundlich para la adsorción de plomo en tallos y en hojas de Eichhornia crassipes, modificadas químicamente.

\begin{tabular}{|c|c|c|c|c|c|c|c|}
\hline \multirow{2}{*}{ Adsorbente } & & \multicolumn{3}{|c|}{ Isoterma de Langmuir } & \multicolumn{3}{c|}{ Isoterma de Freundlich } \\
\cline { 2 - 8 } & $\mathbf{q}(\mathbf{m g} / \mathbf{g})$ & $\mathbf{b} \mathbf{( m g} / \mathbf{L})$ & $\mathbf{r}$ & $\mathbf{R L}$ & $\mathbf{K f} \mathbf{( m g} / \mathbf{g})$ & $\mathbf{n}$ & $\mathbf{r}$ \\
\hline TEC & 172,4138 & 8,8448 & 0,9827 & 0,00185 & 20,9115 & 1,5363 & 0,9640 \\
\hline HEC & 131,5789 & 6,9210 & 0,9823 & 0,00328 & 19,7460 & 1,6273 & 0,9687 \\
\hline
\end{tabular}


Si se comparan las capacidades de adsorción de E. crassipes con las de otros biomateriales que han sido usados para la remoción de plomo, tales como cáscara de yuca y ñame: qmax de 52,34 y 98,36mg/g, respectivamente (Tejada et al. 2016); bagazo de caña: qmax de 162 y 451mg/g para material sin modificar y modificado con ácido cítrico 0,6M, respectivamente (Tejada et al. 2015); carbón activado preparado con semilla de aguaje (Miurita flexuosa): qmax 74,8mg/g (Sun-Kou et al. 2014); Pasto (Pennisetum clandestinum hochst) modificado con $\mathrm{H}_{2} \mathrm{SO}_{4}$ a $1,25 \%$ : qmax $=139,35 \mathrm{mg} / \mathrm{g}$ (Maldonado et al. 2012), se establece que las biomasas TEC y HEC utilizadas en el presente estudio muestran un alto potencial para la remoción de plomo en solución acuosa.
Calcinación de biomasa residual: La curva de la figura 4 muestra que, al aumentar la temperatura de calcinación, la ceniza se hace más resistente a las soluciones ácidas, cuyo $\mathrm{pH}$ condiciona, de igual manera, el comportamiento del material calcinado. En todos los casos, la calcinación estabiliza el material residual, logrando una retención de plomo superior al 98\%, para una concentración inicial de 100ppm; solo un pequeño porcentaje es liberado por efecto del ácido nítrico empleado, para desorber las cenizas.

La acidez de la solución lixiviante afecta la capacidad de retención de los iones, como consecuencia de la alteración en la estabilidad estructural del biomaterial, haciendo que las

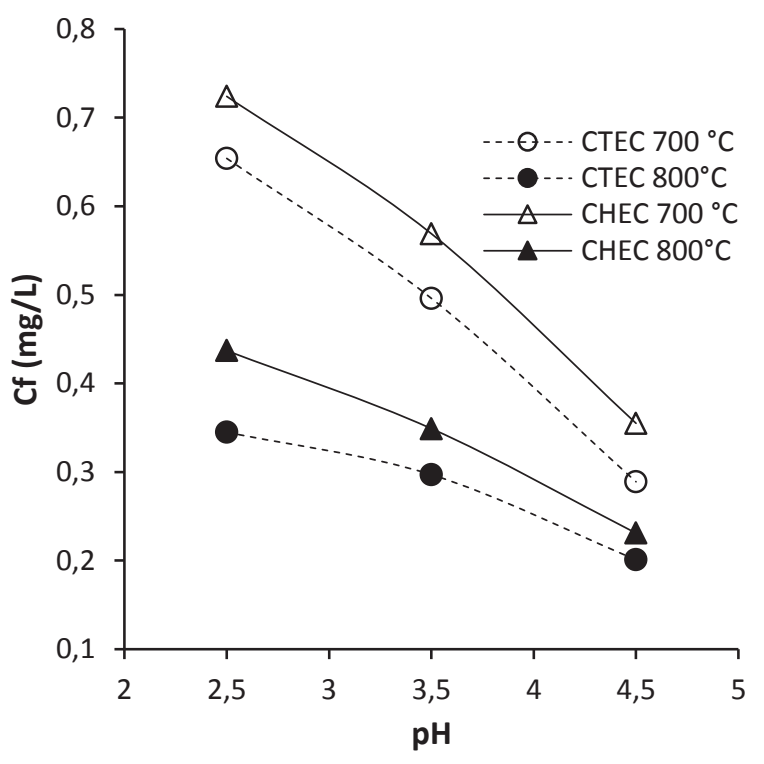

Figura 4. Desorción de plomo en cenizas de tallos (CTEC) y hojas (CHEC) de Eichhornia crassipes.

cadenas formadas entre los sitios activos de la pared celular y los cationes se fragmenten, por efecto de la presencia de iones hidronio $\mathrm{H}_{3} \mathrm{O}^{+}$, con alta movilidad iónica, que compiten con el catión metálico (Navarro et al. 2006).

El comportamiento de las cenizas de TEC y HEC es similar a los reportados por Vizcaino \& Fuentes (2015), quienes en ensayos de calcinación de algas rojas, cáscara de naranja y tuna, bajo condiciones similares de temperatura y de tiempo, obtuvieron una retención de plomo superior al 95\%.

Los resultados del proceso de desorción indican que el biomaterial constituye una alternativa no solo para el proceso de eliminación del contaminante sino, además, para su almacenamiento y desactivación.
Basado en los resultados obtenidos en la investigación, se concluye que $E$. crassipes es una especie con gran capacidad para la fitorremediacion de efluentes residuales contaminados con plomo. La matriz biológica presente en los tallos y las hojas de $E$. crassipes captura los iones $\mathrm{Pb}^{+2}$, impidiendo su liberación y su movilidad, aun al someterla al ataque de soluciones con pH altamente ácidos, constituyéndola en una alternativa viable y económica para la descontaminación de aguas, con concentraciones de plomo $\leq 100 \mathrm{ppm}$.

Se recomienda realizar ensayos para evaluar la capacidad de regeneración de $E$. crassipes y su rendimiento en un segundo ciclo de biosorción, con el propósito de reutilizar el material biosorbente. Asimismo, se hace necesario realizar ensayos en sistemas de flujo continuo, que permitan establecer los tiempos de ruptura y saturación a diferentes velocidades 
de flujo volumétrico, a fin de describir el comportamiento dinámico de las condiciones de operación y el rendimiento de la columna.

Agradecimientos: Los autores expresan su agradecimiento a la Universidad de La Guajira sede Fonseca y a los auxiliares de investigación Emerson Brito, Jain Duran y Gustavo Mejía, por su apoyo y compromiso en las fases de campo y laboratorio. Conflictos de intereses: El manuscrito fue preparado y revisado con la participación de los autores, quienes declaramos que no existe conflicto de intereses que ponga en riesgo la validez de los resultados presentados. Financiación: Esta investigación fue desarrollada con los recursos financieros aportados por los autores y con recursos técnicos aportados por la Universidad de La Guajira.

\section{BIBLIOGRAFÍA}

1. ABDEL-GHANI, N.; EL-CHAGHABY, G. 2014. Biosorption for metal ions removal from aqueous solution: A review of recent studies. Int. J. Latest Res. Scienc. Techn. 3(1):24-42.

2. ATEHORTÚA, E.; GARTNER, C. 2013. Estudios preliminares de la biomasa seca de Eichhornia crassipes como adsorbente de plomo y cromo en aguas. Rev. Col. Materiales. 4:81-92.

3. CÁRDENAS, J.; MOCTEZUMA, M.; ACOSTA, I.; MARTÍNEZ, V. 2013. Biosorción de Plomo (II) en solución por diferentes biomasas fungicas. Rev. Latinoam. Recursos Naturales (México). 9(1):57-61.

4. CHUQUILIN, R.; ROSALES, D. 2016. Estudio de la biosorción de Cd (II) Y Pb (II) usando como adsorbente Nostoc sphaericum Vaucher. Rev. Soc. Quím. Perú. 81(1):49-60.

5. CUIZANO, N.; NAVARRO, A. 2008. Biosorcion de metales pesados: Posible solución a la contaminacion a bajas concentraciones. An. Quim.(España). 104(2):120-125.

6. DELGADILLO, A.; GONZALEZ, C.; PRIETO, F.; VILLAGOMEZ, J.; ACEVEDO, O. 2011. Fitorremediación: una alternativa para eliminar la contaminación. Trop. subtrop. agroecosyt, 14(2): 597-612.

7. DÍAZ, A.; ARIAS, J.; GELVES, G.; MALDONADO, A.; LAVERDE, D.; PEDRAZA, J.; ESCALANTE, H. 2003. Biosorción de $\mathrm{Fe}, \mathrm{Al}$ y $\mathrm{Mn}$ de drenajes ácidos de mina de carbón empleando algas marinas Sargassum sp. en procesos continuos. Rev. Fac. Ingenieria UdeA. (Colombia). (30):34-48.
8. GARCÍA, V.; BORJA, N.; GUZMAN, E.; YIPMANTIN, A.; MALDONADO, H. 2013. equilibrio de biosorción de plomo (II) y caracterizacion mediante FT-IR y SEMEDAX en pectina reticulada proveniente de cáscaras de naranja. Rev. Soc. Quím. Perú. 79(3):256-264.

9. GUO, X.; SHAN, X.; ZHANG, S. 2007. Adsorption of metal ions on lignin. J. Hazardous Materials. 151(1):134142.

10. LAVADO, C.; SUN KOU, M.; BENDEZÚ, S. 2010. Adsorción de plomo de efluentes industriales usando carbones activados con $\mathrm{H}_{3} \mathrm{PO}_{4}$. Rev. Soc. Quím. Perú. 76(2):165-178. Disponible desde Internet en: http:// www.redalyc.org/pdf/3719/371937617008.pdf (con acceso 25/07/2017).

11. LODEIRO, P.; BARRIADA, J.; HERRERO, R.; SASTRE DE VICENTE, M. 2006. The marine macroalga Cystoseira baccata as biosorbent for caadmium(II) and lead(II) removal: Kinetic and equilibrium studies. Environmental Pollution. 142(2):264-273. Disponible desde Internet en: https://doi.org/10.1016/j.envpol.2005.10.001 (con acceso 25/07/2017).

12. MALDONADO, A.; LUQUE, C.; URQUIZO, D. 2012. Biosorcion de plomo de aguas contaminadas utilizando Pennisetum clandestinum Hochst (kikuyo). Rev. Latinoam. Metal. Mat (Venezuela). 4:52-57.

13. MCCABE, W.; SMITH, J.; HARRIOTT, P. 1998. Operaciones Unitarias en ingenieria quimica. (Cuarta edicion ed.). Madrid (España): Mc Graw Hill. 1095p.

14. MURITHI, G.; ONINDO, C.; WAMBU, E.W.; MUTHAKIAC, G.K. 2014. Removal of Cadmium(II) Ions from Water by Adsorption using Water Hyacinth (Eichhornia crassipes) Biomass. BioResources. 9(2):3613-3631.

15. NAVARRO, A.; RAMOS, K.; CAMPOS, K.; MALDONADO, H. 2006. Elucidacion del efecto del pH en la adsorción de metales pesados mediante biopolimeros naturales: Cationes divalentes y superficies activas. Rev. Iberoam. Polímeros (País Vasco). 7(2):113-126. Disponible desde Internet en: http://www.reviberpol. iibcaudo.com.ve/pdf/MAY06/navarro.pdf (con acceso $25 / 07 / 2017$ ).

16. ÓRE, F.; LAVADO, C.; BENDEZU, S. 2015. Biosorción de $\mathrm{Pb}$ (II) de aguas residuales de mina usando el marlo de maiz (Zea mays). Rev Soc. Quím. Perú. 81(2):122-134. 
17. RAMÍREZ, J.; ENRÍQUEZ, M. 2015. Remoción de plomo (II) usando lignina obtenida a partir del procesamiento del seudotallo de plátano. Acta Agronómica. 64(3):209-213.

18. RUBIO, C.; GUTIÉRREZ, A.; MARTÍN IZQUIERDO, R.; REVERT, C.; LOZANO, G.; HARDISSON, A. 2004. El plomo como contaminante alimentario. Rev. Toxicología (España). 21:72-80. Disponible desde Internet en: http://www.fmed.uba.ar/depto/toxico1/articulos/7.pdf (con acceso 25/07/2017).

19. SUIN-KOU, M.; OBREGON, D.; PINEDO, A.; PAREDES, A.; AYLAS, J. 2014. Adsorción de metales pesados empleando carbones activados preparados a partir de semillas de aguaje. Rev Soc Quím Perú. 80(4):225-236.

20. TEJADA, C.; MONTIEL, Z.; ACEVEDO, D. 2016. Aprovechamiento de cáscaras de yuca y ñame para el tratamiento de aguas residuales contaminadas con $\mathrm{Pb}$ (II). Inf. tecnol (Chile). 27(1):9-20. Disponible desde Internet en: http://www.scielo.cl/pdf/infotec/ v27n1/art03.pdf (con acceso 25/07/2017).

21. TEJADA, C.; RUIZ, E.; GALLO, J.; MOSCOTE, J. 2015. Evaluación de la biosorción con bagazo de palma africana para la eliminación de $\mathrm{Pb}(\mathrm{II})$ en solución. Prospect. (Colombia). 13(1):59-67. Disponible desde Internet en: http://dx.doi.org/10.15665/rp.v13i1.360 (con acceso 25/07/2017).

22. TELLO, W.; SALVATIERRA, L.; PÉREZ, L. 2015. Evaluación de los mecanismos de eliminación de $\mathrm{Pb}^{2+}$ en sistemas de fitorremediación en lotes operados con Salvinia biloba raddi (acordeón de agua). Energeia. 13(13): 10-17.

23. VERA, K.; RAMOS, K.; CAMARGO, E.; ANDRADE, C.; NUÑEZ, M.; DELGADO, J.; MORALES, E. 2016. Fitorremediación de aguas residuales con alto contenido de plomo utilizando Typha dominguensis y Canna generalis. Rev. Téc. Ing. Univ. Zulia. 39(2):88-95.

24. VIZCAINO, L.; FUENTES, N. 2015. Biosorción de Cd, Pb y Zn por biomasa pretratada de algas roja, cáscara de naranja y tuna. Ciencia Ingeniería Neogranadina (Colombia). 25(1):43-60.

Recibido: Febrero 27 de 2017

Aceptado: Nov. 19 de 2017

\section{Cómo citar:}

Vizcaíno Mendoza, L.; Fuentes Molina, N.; González Fragozo, H. 2017. Adsorción de plomo (II) en solución acuosa con tallos y hojas de Eichhornia crassipes. Rev. U.D.C.A Act. \& Div. Cient. 20(2): 435-444. 